\title{
Absolute Calibration of the National Bureau of Standards Photoneutron Standard: I
}

\author{
J. A. De Juren, D. W. Padgett, and L. F. Curtiss
}

\begin{abstract}
The absolute emission rate of neutrons from the National Bureau of Standards radiumberyllium photoneutron standard source has been determined from the volume integral of the neutron absorption rate in water. Thin indium and manganese foils were employed to measure the thermal-neutron density as a function of distance from the source. The foil activities were converted to neutron densities by calibration of the foils in a reference thermalneutron flux previously calibrated with boron films. A motor driven mechanical integrator, which moves foils radially from the source at a rate that takes into account the increase in area with distance from the source and the decay of the foils, provided over 80 percent of the volume integral. A value of $1.26_{5} \times 10^{6}$ neutrons per second was determined for the emission rate of the photoneutron standard, with an estimated standard error of 3 percent.
\end{abstract}

\section{Introduction}

It is desirable that a neutron standard have a nearly constant rate of emission over a relatively long period of years, or that corrections due to changes follow some law which makes their computation simple. $\operatorname{Ra}-\operatorname{Be}(\alpha, n)$ sources do not fulfill this requirement since their strength depends upon other factors than the amounts of radium and beryllium present, and because the growth of the alphaparticle emitting polonium causes an increase in the neutron emission rate of approximately 9 percent at equilibrium. Therefore, a $\operatorname{Ra}-\operatorname{Be}(\gamma, n)$ source was selected as a standard because its decrease in activity with time is proportional to the decrease of radium.

Two nearly identical radium-beryllium photoneutron sources $[1]^{1}$ have been constructed to serve as standards for the Bureau. Each source consists of a beryllium sphere, $4.0 \mathrm{~cm}$ in diameter, at the center of which is placed one curie of radium in the form of $\mathrm{RaBr}_{2}$. The radium in both sources was initially enclosed in platinum-iridium capsules of $0.2 \mathrm{~mm}$ wall thickness. One capsule developed a leak and was subsequently enclosed in monel of 1 $\mathrm{mm}$ thickness. ${ }^{2}$ The source having the original capsule (NBS \#1) has been chosen as the primary standard for the absolute calibration, and an aluminum jacket was placed around the beryllium to protect it.

The calibration of the neutron emission rate is based on the Frisch-Walker method [2] with certain modifications.

\section{Method of Calibration}

In this method of calibration the source is placed in a volume filled with a moderating medium which slows down and captures practically all neutrons as thermal neutrons before they escape from the volume. Then the absolute source strength, $Q$, in number of neutrons emitted per second is directly equal to the neutron capture rate per second. This rate is de-

\footnotetext{
1 Figures in brackets indicate the literature references at the end of this paper. ${ }_{2}$ This source may be borrowed by other laboratories for comparison measurements.
}

termined, in principle, by measuring the density of thermal neutrons at various points in the volume, converting this to rate of capture by means of the thermal capture cross section for the medium, and integrating over the whole volume.

In the measurements to be reported, water was used as the moderating medium. As detectors of thermal neutrons thin indium or manganese foils are used at various radial distances, $r$, from the source and acquire a certain saturated activity, $A_{\mathrm{th}}(r)$, after exposure in the medium. Consequently these foils must be calibrated to permit the measured activity to be interpreted in terms of thermal-neutron density. Nearly all captures of neutrons in the water occur at energies less than the cadmium cutoff $(0.5 \mathrm{ev})$, and indium and manganese have neutron capture cross sections which vary approximately as $1 / v$ in the thermal region. A reference flux, calibrated with boron films exposed in small pulse ionization chambers, was used to calibrate the foils. For the same foil activity in both the reference flux and water the ratio of the capture rate per mole of boron to the capture rate per mole of hydrogen is proportional to the ratio of the capture cross sections, $\sigma_{\mathrm{B}} / \sigma_{\mathrm{H}}$.

The calibration is carried out in such a manner that the absolute values of the cross sections of boron and hydrogen need not be known. The number of neutrons per $\mathrm{cm}^{3} / \mathrm{sec}$ captured in the thermal region as a function of the distance, $r$, from the center of the source is given by $n(r) v M_{\mathrm{H}} \sigma_{\mathrm{H}}$ where $n(r)$ is the thermal-neutron density, $v$ the neutron velocity, $M_{\mathrm{H}}$ the concentration of hydrogen in moles per cubic centimeter, and $\sigma_{\mathrm{H}}$ the thermal-neutron absorption cross section per mole of hydrogen. If the neutrons captured at energies greater than the cadmium cutoff be expressed as a fraction $\epsilon(r)$ of the number captured in the thermal region then

$$
Q=4 \pi \int_{a}^{\infty} n(r) v M_{\mathrm{H}} \sigma_{\mathrm{H}}[1+\epsilon(r)] r^{2} d r
$$

where $a$ is the radius of the source and no self-absorption is assumed. A small correction will be made 
later for neutron absorption in the source. As $\sigma_{\mathrm{H}} v$ is a constant over the thermal region it may be removed from the integral and

$$
Q=4 \pi M_{\mathrm{H}} \sigma_{\mathrm{H}} v \int_{a}^{\infty} n(r)[1+\epsilon(r)] r^{2} d r .
$$

The neutron density, $n(r)$ is obtained by use of a reference thermal neutron flux having a thermal neutron density, $n_{\mathrm{B}}$. A measured amount of boron is exposed in a reproducible position of the flux and the disintegration rate per mole, $I_{\mathrm{B}}$, of the boron measured by counting alpha particles from the reaction $\mathrm{B}^{10}(n, \alpha) \mathrm{Li}^{7}$ with a pulse ionization chamber or a proportional counter. The detector foil is arranged to sample the same flux as the boron and a saturated activity, $A_{\mathrm{B}}$, is measured. Then

$$
\frac{A_{\mathrm{B}}}{A_{\mathrm{th}}(r)}=\frac{n_{\mathrm{B}}}{n(r)} \text {. }
$$

But

$$
I_{\mathrm{B}}=n_{\mathrm{B}} v \sigma_{\mathrm{B}} \text { or } n_{\mathrm{B}}=\frac{I_{\mathrm{B}}}{v \sigma_{\mathrm{B}}}
$$

which gives when inserted in eq (2)

$$
n(r)=\frac{A_{\mathrm{th}}(r) I_{\mathrm{B}}}{A_{\mathrm{B}} v \sigma_{\mathrm{B}}}
$$

Introducing this expression for $n(r)$ into eq (1b) we have

$$
Q=4 \pi \frac{I_{\mathrm{B}}}{A_{\mathrm{B}}} M_{\mathrm{H}} \frac{\sigma_{\mathrm{H}}}{\sigma_{\mathrm{B}}} \int_{a}^{\infty} A_{\mathrm{th}}(r)[1+\epsilon(r)] r^{2} d r
$$

which expresses $Q$ in terms of the integral of the saturated activity of the foils (with epithermal correction term) multiplied by an expression containing the readily measured constants: $I_{\mathrm{B}}, A_{\mathrm{B}}$, and $M_{\mathrm{H}}$ and the ratio $\sigma_{\mathrm{H}} / \sigma_{\mathrm{B}}$ yet to be determined.

\section{Experimental Procedure}

$I_{\mathrm{B}}$ was measured by exposing thin boron films of known mass in pulse-ionization chambers in the NBS thermal-neutron flux [3] generated by two $\mathrm{Ra}-\mathrm{Be}(\alpha, n)$ sources imbedded in a moderator. This calibration yielded a value of $I_{\mathrm{B}}=1.76_{1} \times 10^{6}( \pm 2 \%$ standard error) disintegrations per second mole. The value of $A_{\mathrm{B}}$, the activity of the foil in this reference flux was measured in the same way, to be described later, as the activity of the foil when exposed in the presence of the source under calibration.

In earlier experiments, $\sigma_{\mathrm{B}} / \sigma_{\mathrm{H}}$ was determined by foil measurements with water and with boric acid solutions as the absorbing media. Unfortunately the boron in the solution may not have the same isotopic composition as the boron used in determining $I_{\mathrm{B}}$. Thode [4] has noted a geographical variation in the isotopic composition of boron and Walker reported a reproducible difference in the counting rate in a counter filled to the same pressure with $\mathrm{BF}_{3}$ generated from two different compounds, when exposed to the same neutron flux.
The boron in the films used for the determination of $I_{\mathrm{B}}$, was compared with the "standard" boron of the Argonne National Laboratory by the danger coefficient method [5]. Since $\sigma_{\mathrm{B}} / \sigma_{\mathrm{H}}$ has been precisely measured for the "standard" boron, the comparison by the danger coefficient method yields $\sigma_{\mathrm{B}} / \sigma_{\mathrm{H}}$ for the NBS boron.

The ratio, $\sigma_{\mathrm{B}} / \sigma_{\mathrm{H}}$ has been measured as $2297 \pm 27$ by Argonne National Laboratory for their "standard" boron [6]. From the danger coefficient comparison

$$
\frac{\sigma_{\mathrm{B}(\mathrm{NBS})}}{\sigma_{\mathrm{B}(\mathrm{Argonne})}}=0.988 \pm 0.006
$$

which gives

$$
\left(\frac{\sigma_{\mathrm{B}}}{\sigma_{\mathrm{H}}}\right)_{\mathrm{NBS}}=226_{4}( \pm 1.3 \%) .
$$

Because water alone was used for the absorbing medium only one comparison of the NBS boron with the "standard" boron is required.

Equation (5a) may be expressed in the following form:

$$
Q=4 \pi \frac{I_{\mathrm{B}}}{A_{\mathrm{B}}} M_{\mathrm{H}} \frac{\sigma_{\mathrm{H}}}{\sigma_{\mathrm{B}}}\left[1+\epsilon_{\mathrm{ave}}\right] \int_{a}^{\infty} A_{\mathrm{th}}(r) r^{2} d r
$$

since

$$
\epsilon_{\mathrm{ave}}=\frac{\int_{a}^{\infty} A_{\mathrm{th}}(r) \epsilon(r) r^{2} d r}{\int_{a}^{\infty} A_{\mathrm{th}}(r) r^{2} d r} .
$$

The ratio of epithermal to thermal captures, $\epsilon(r)$, is evaluated by measurements with a small boron-trifluoride or boron-lined counter, with and without a cadmium cover, as a function of distance from the source. From earlier measurements in our laboratory and by Walker $\epsilon_{\text {ave }}=0.008 \pm 0.001$ for pure water.

We now proceed to describe the method of evaluating the integral, $4 \pi \int_{a}^{\infty} A_{\mathrm{th}}(r) r^{2} d r$, appearing in the formula for $Q$. To permit the use of a mechanical integrator in the experiment for determining the major part of this integral, the region was broken into three spherical shells having radii extending (1) from $r=5.0 \mathrm{~cm}$ to $r=20.0 \mathrm{~cm}$, (2) from $r=20.0 \mathrm{~cm}$ to $r=\infty$, and (3) from $r=2.1 \mathrm{~cm}$ to $r=5.0 \mathrm{~cm}$. The addition of the contributions to the integral from these three regions gives the value from $r=2.1 \mathrm{~cm}$ to $r=\infty$.

In the experimental arrangement the source was pin-mounted on a stainless steel rod in the center of the water which filled a stainless steel tank $107 \mathrm{~cm}$ in diameter to a depth of $100 \mathrm{~cm}$ as shown in figure 1. The four detecting foils were held in light aluminum frames by bifilar suspension from a metal framework above the tank. Lead weights were suspended at some distance below the frames, which were centered in a horizontal plane passing through the center of the source at positions $90^{\circ}$ apart. A 


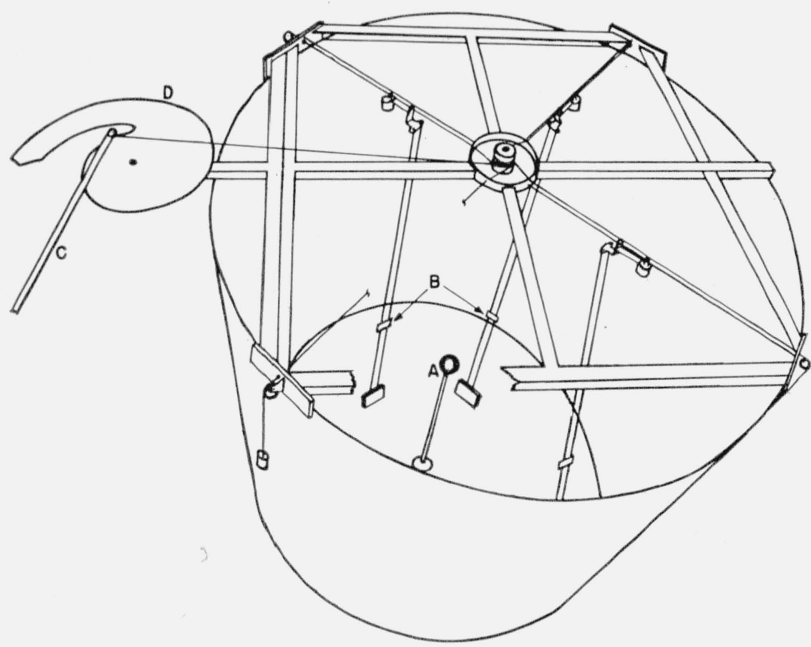

Figure 1. Neutron integration system. A, Photoneutron source; B, foils; C, follower arm; D, cam.

cathetometer mounted, with scale horizontal, above the tank permitted the foils to be set within a fraction of a millimeter of the desired radius from the source. The distance from the center of the source to the center of the foil was chosen to give the desired root mean square distance averaged over the finite area of the foil. In water over 98 percent of the neutrons from the photoneutron standard are absorbed within $20 \mathrm{~cm}$ from the center of the source. Normally a series of measurements would be made of foil activities as a function of distance from the source and the integral

$$
4 \pi \int_{a}^{\infty} A_{\mathrm{th}}(r) r^{2} d r
$$

obtained by graphical integration. This procedure has been followed in the marginal regions (2) and (3) but most of the integral has been obtained by a faster method. Following the method of Frisch and Walker, a mechanical integrator was used to determine the foil integral in the region from 5.0 to $20.0 \mathrm{~cm}$. This integrator moves the foils at a rate to take into account the $r^{2}$ increase in area and subsequent decay of the induced activity as the range is covered. In the interest of completeness the derivation of the Frisch-Walker cam equations is given in the appendix.

\subsection{Evaluation of Integral From Radius of Source to $r=5.0 \mathrm{~cm}$}

Slowing down theory indicates that the thermal neutron density distribution near the source is approximately gaussian and may be represented by the equation

$$
A_{\text {th }}(r)=C e^{-r^{2} / r_{0}^{2}}
$$

The constants $C$ and $r_{0}$ were initially evaluated by indium foil activities at 4.5 and $5.0 \mathrm{~cm}$. As about 15 percent of the integral lies within $5.0 \mathrm{~cm}$, more

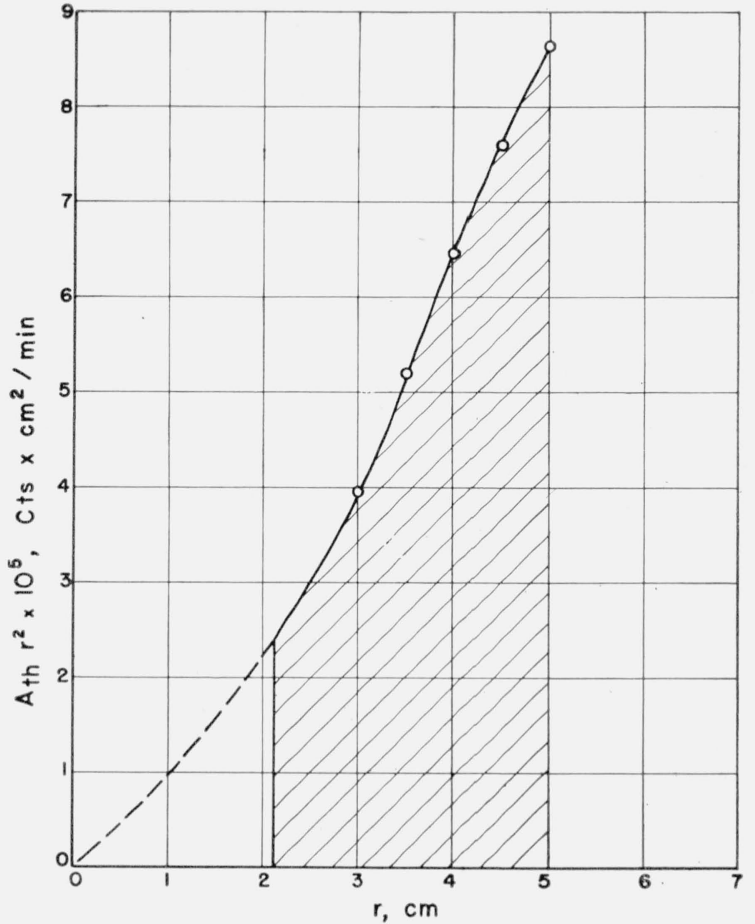

Figure 2. Thermal integral for first set of indium foils.

$$
4 \pi \int_{2.1}^{5.0} A_{\text {th }} r^{2} d r=19.71 \times 10^{6} \mathrm{cts} \times \mathrm{cm}^{3} / \mathrm{min} .
$$

extensive measurements closer to the source were taken with manganese foils $1 \mathrm{~cm}^{2}$ and 3 mil thick, at $0.5 \mathrm{~cm}$ intervals from 3.0 to $5.0 \mathrm{~cm}$. The manganese activities were normalized to the indium foil measurements by the ratio of the indium to manganese thermal activities at $5.0 \mathrm{~cm}$ and graphically integrated from 3.0 to $5.0 \mathrm{~cm}$. A plot of $A_{\mathrm{th}}(r) r^{2}$ as a function of $r$ for the normalized manganese foils is given in figure 2 .

\subsection{Extrapolation to $r=\infty$}

As the maximum neutron energy from the photoneutron standard is only $0.7 \mathrm{Mev}$, thermalization occurs close to the source in water and beyond $20 \mathrm{~cm}$ the neutron density distribution is almost entirely determined by the diffusion of thermal neutrons. For any source if the relaxation length in the surrounding medium is less than the diffusion length, diffusion will dominate at large radii. This principle was used to measure the diffusion length of thermal neutrons in water [7] and a value $L=2.763$ $\pm 0.015 \mathrm{~cm}$ was obtained.

Since the neutron density results from thermal diffusion,

$$
\begin{gathered}
A_{\mathrm{th}}(r)=(B / r) e^{-r / L} \\
4 \pi \int_{20}^{\infty} A_{\text {th }}(r) r^{2} d r=4 \pi B \int_{20}^{\infty} r e^{-\tau / L} d r .
\end{gathered}
$$


Evaluating $B$ at $r=20.0 \mathrm{~cm}$

$$
B=20 A_{\text {th }}(20) e^{20 / L} .
$$

In terms of the activity at $20 \mathrm{~cm}$ the integral becomes

$$
\begin{aligned}
4 \pi \int_{20}^{\infty} A_{\text {th }}(r) r^{2} d r & \\
& =80 \pi A_{\text {th }}(20) L[20+L] \\
& =1.580 \times 10^{4} A_{\text {th }}(20) .
\end{aligned}
$$

\section{Measurement of Foil Âctivities}

The first series of measurements were taken with 20 percent indium 80 percent tin foils, $2.0 \times 3.4 \mathrm{~cm}^{2}$ in area and 0.003 in. thick. Four foils were irradiated simultaneously at the position desired and then counted with thin mica end-window Geiger counters whose outputs were fed to a mixing circuit so that the activities could be summed. Individual counting rates were close enough so that the statistical weights were about the same for each foil. Uranium standards were used to check the constancy of the counters. An average resolving time of 270 $( \pm 10 \%) \mu$ sec, determined by the two source method, was used for the individual counters. For the highest counting rates the resolving time correction was less than 5 percent. In the determination of $A_{B}$ the foils were counted successively after each was irradiated alone in the standard pulse chamber in the position occupied by the boron film.

Normally exposures were made overnight and the saturated foil activity was determined from repetitive cycles of foils, uranium standards, and background using a value of $54.0 \mathrm{~min}$ for the indium half-life.

The first series of foil measurements were made in 1951-2. A second set of measurements was taken early in 1954 after the standards laboratory was transferred to another building.

Only two foils $180^{\circ}$ apart could be followed by the cathetometer when the cam was being used. Any slight displacement of the source perpendicular to the line of centers of the foils causes an error of the second order in the distance from source to foil. For the above reasons two rather than four foils were employed in the second series of measurements. A flow counter operating in the proportional region counted the foils which were exposed internally beneath the anode wire. No monitor was required with this counter. The resolving time of the counter and associated electronics is less than $5 \mu$ sec and the loss at the highest counting rate is less than 0.3 percent. During the first series of measurements 0.020 in. cadmium covers were placed around the aluminum foil holders when indium resonance irradiations were taken in the water tank. As a result about $0.5 \mathrm{~cm}$ thickness of water was within the cadmium covers and part of the activity resulted from thermalization within this volume in addition to the $1.44 \mathrm{ev}$ indium resonance neutrons. In the second measurements the cadmium covers fit tightly about the foils so this effect could not occur. Irradiations were also made with cadmium around the aluminum frames so a correction could be applied to the initial measurements.

\subsection{Neutron Depression}

A correction must be made for the local depression in neutron density caused by a foil when placed in the water. Measurements taken here and elsewhere [8] indicate that empirical modifications of Bothe's formulae give a close fit to the neutron depression caused by absorbing foils in various media. The formulae for the factor, $F_{\text {th }}$, by which the foil activities must be multiplied to correct for the depression are

$$
\begin{aligned}
& F_{\mathrm{th}}=1+\frac{\alpha}{2}\left[\frac{3 R}{2 \lambda_{\mathrm{tr}}} \frac{L}{R+L}-1\right] \text { if } R \gg \lambda_{\mathrm{tr}} \\
& F_{\mathrm{th}}=1+\frac{0.34 \alpha R}{\lambda_{\mathrm{tr}}} \text { if } R \ll \lambda_{\mathrm{tr}}
\end{aligned}
$$

where $\alpha$ is the average probability of absorption of a neutron in an isotropic flux by a circular foil of radius $R, L$ is the diffusion length, and $\lambda_{\text {tr }}$ the transport mean free path in the diffusive medium.

$$
\begin{aligned}
\alpha & =1-e^{-t}(1-t)+t^{2} E I(-t) \\
t & =\frac{\rho x \bar{\sigma} N_{0}}{M}
\end{aligned}
$$

where $\rho x$ is the thickness of the foil in grams per square centimeter, $M$, the atomic weight, $\bar{\sigma}$ the absorption cross section at the mean velocity, and $N_{0}$ is Avogadro's number,

$$
E I(-t)=\int_{t}^{\infty} \frac{e^{-t}}{t} d t .
$$

For the first set of foils using

$$
\begin{aligned}
\pi R^{2} & =6.8 \mathrm{~cm}^{2}, \\
\rho X & =10.08 \mathrm{mg} / \mathrm{cm}^{2} \text { of indium, } \\
\bar{\sigma} & =171 \mathrm{barns}, \\
\lambda_{\mathrm{tr}} & =0.425 \mathrm{~cm}, \\
L & =2.76 \mathrm{~cm}, \\
F & =1+0.0126 \text { using eq }(9), \text { and } \\
F & =1+0.0124 \text { using eq }(10) .
\end{aligned}
$$

One of the foils used in the 1954 measurements was only 2.5 mils thick and $F=1.012$ for the second set from eq (9). In the standard chamber the depression is negligible. A correction must also be made for the absorption of indium resonance neutrons by the cadmium covers. Earlier measurements made of the decrease of foil activity with increasing thickness of 
cadmium yielded a value of 1.04 for the correction factor with $0.020 \mathrm{in}$. cadmium covers. This correction introduces only a slight error if neglected since it occurs in the ratio of the cadmium difference activities.

\section{Data}

The foil activities at saturation are given in tables 1 and 2. A correction has been applied to the resonance activities measured with the first set of

TABLE 1. Foils 1, 2, 3, and 4 counted collectively with end window Geiger tubes

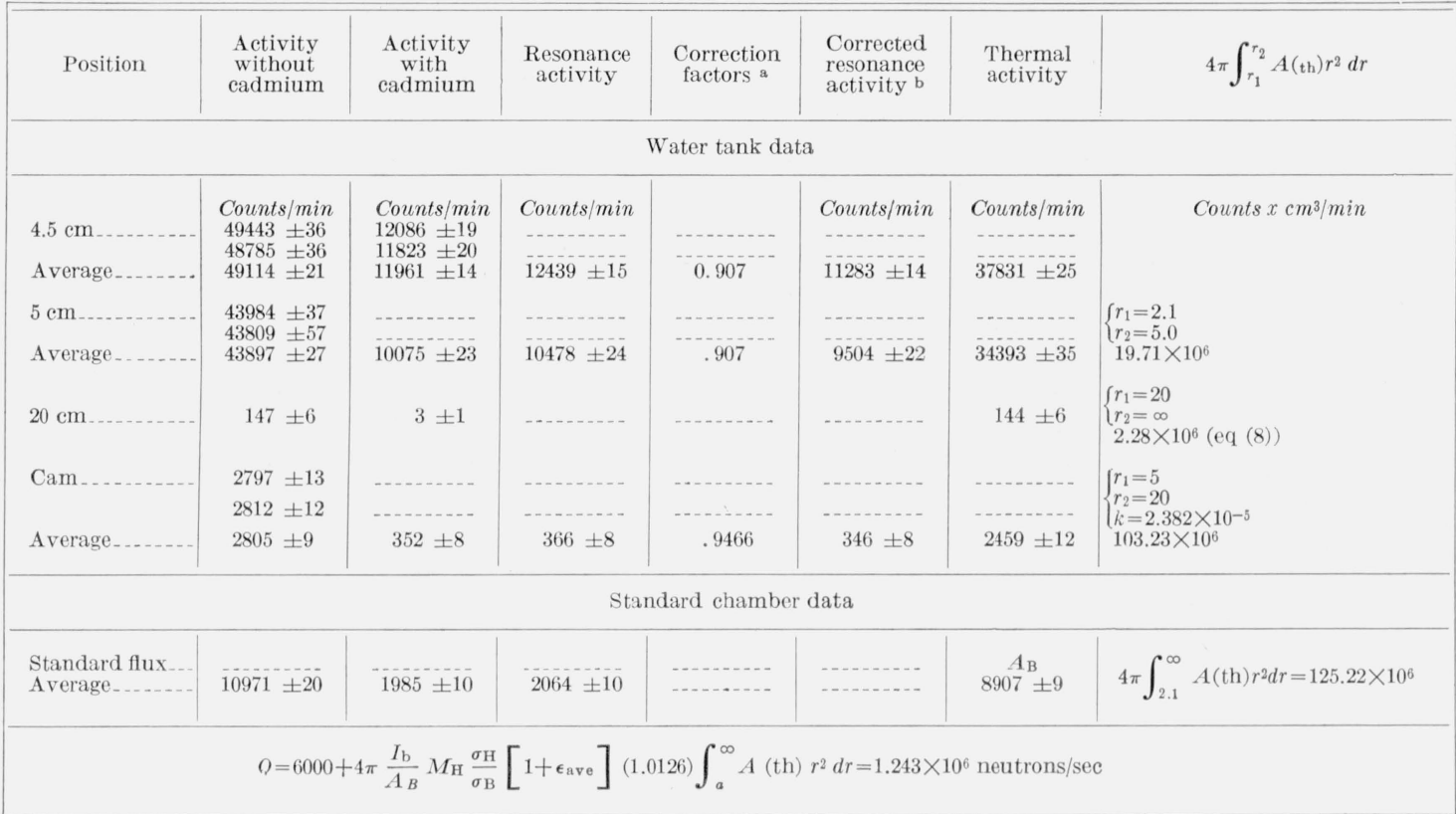

a Correction factors were applied for the thermalization within the cadmium covers.

b Resonance activity $=1.04$ Xactivity with cadmium.

TABLE 2. Foils 5 and 6 counted with flow proportional counter

\begin{tabular}{|c|c|c|c|c|c|c|}
\hline Position & $\begin{array}{l}\text { Activity } \\
\text { without } \\
\text { cadmium }\end{array}$ & $\begin{array}{l}\text { Activity } \\
\text { with } \\
\text { cadmium }\end{array}$ & $\begin{array}{l}\text { Resonance a } \\
\text { activity }\end{array}$ & $\begin{array}{l}\text { Thermal } \\
\text { activity }\end{array}$ & $4 \pi \int_{r_{1}}^{r_{2}} A_{\mathrm{th}} r^{2} d r$ & $\begin{array}{l}\text { Thermal } \\
\text { capture }\end{array}$ \\
\hline \multicolumn{7}{|c|}{ Water tank data } \\
\hline \multirow[t]{2}{*}{$5 \mathrm{~cm}$} & $\begin{array}{l}\text { Counts/min } \\
38134 \pm 40\end{array}$ & $\begin{array}{l}\text { Counts/min } \\
8050 \pm 14\end{array}$ & Counts/min & Counts/min & Counts $x \mathrm{~cm}^{3} / \mathrm{min}$ & $\%$ \\
\hline & $38023 \pm 55$ & $8072 \pm 14$ & - & -non- & $\left\{\begin{array}{l}r_{1}=2.1 \\
r_{2}=5.0\end{array}\right.$ & \}$\cdots$ \\
\hline A verage.......... & $38096 \pm 32$ & $8061 \pm 10$ & $8383 \pm 10$ & $29713 \pm 34$ & $16.92 \times 10^{6}$ & 15.7 \\
\hline $20 \mathrm{~cm}$ & $\begin{array}{l}119.8 \pm 3.4 \\
119.1 \pm 3.3\end{array}$ & (n) & - n- & - & $\left\{\begin{array}{l}r_{1}=20 \\
r_{2}=\infty\end{array}\right.$ & \} \\
\hline A verage......... & $119.4 \pm 2.4$ & $3 \pm 1$ & (n) & $116 \pm 3$ & $1.83 \times 10^{6}$ & 1.7 \\
\hline Cam & $2423 \pm 8$ & $292.0 \pm 3.7$ & - & (n) & $r_{1}=5$ & \\
\hline A verage.......... & $\begin{array}{l}2439 \pm 8 \\
2431 \pm 6\end{array}$ & $\begin{array}{c}289.5 \pm 4.0 \\
291 \pm 3\end{array}$ & $302 \pm 3$ & $2129 \pm 7$ & $\left\{\begin{array}{l}\left\{\begin{array}{l}89.37 \times 10^{6} \\
r_{2}=5\end{array}\right. \\
\frac{-0.45 \times 10^{6}}{88.92 \times 10^{6}} \text { cam correction }\end{array}\right.$ & 82.6 \\
\hline & & & & & \multicolumn{2}{|c|}{$4 \pi \int_{2.1}^{\infty} A_{\mathrm{th}} r^{2} d r=107.67 \times 10^{6}$} \\
\hline \multicolumn{7}{|c|}{ Standard chamber data } \\
\hline $\begin{array}{l}\text { Standard flux... } \\
\text { A rerage.......... }\end{array}$ & $\begin{array}{l}9293 \pm 21 \\
9300 \pm 14 \\
9298 \pm 12\end{array}$ & $\begin{array}{l}1709 \pm 7 \\
1716 \pm 10 \\
1711 \pm 6\end{array}$ & $1780 \pm 6$ & $7518 \pm 13$ & - & 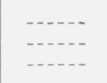 \\
\hline
\end{tabular}

a Resonance activity $=1.04$ Xactivity with cadmium. 
TABLE 3. Foil activities within $5 \mathrm{~cm}$ of source in water

\begin{tabular}{|c|c|c|c|c|c|c|}
\hline Foils & $r$ & $\begin{array}{c}\text { Activity } \\
\text { without } \\
\text { cadmium }\end{array}$ & $\begin{array}{l}\text { Activity } \\
\text { with } \\
\text { cadmium }\end{array}$ & $\begin{array}{l}\text { Resonance } \\
\text { activity }\end{array}$ & $\begin{array}{l}\text { Thermal } \\
\text { activity }\end{array}$ & $4 \pi \int_{2.1}^{5 \mathrm{em}} A_{\mathrm{th}} r^{2} d r$ \\
\hline Indium set I.... & $\begin{array}{l}\mathrm{cm} \\
4.5 \\
5.0\end{array}$ & $\begin{array}{l}\text { Counts/min } \\
49114 \pm 21 \\
43897 \pm 27\end{array}$ & $\begin{array}{c}\text { Counts/min } \\
10849 \pm 13.5 \\
9138 \pm 22\end{array}$ & $\begin{array}{c}\text { Counts/min } \\
11283 \pm 14 \\
9504 \pm 23\end{array}$ & $\begin{array}{l}\text { Counts/min } \\
37831 \pm 25 \\
34393 \pm 35\end{array}$ & $\begin{array}{r}\text { Counts } \times \mathrm{cm}^{3} / \mathrm{min} \\
2.009 \times 10^{7} \mathrm{Gaussian} \text { fit }\end{array}$ \\
\hline $\begin{array}{l}\text { Manganese a } \\
1 \mathrm{~cm} \times 1 \mathrm{~cm} \times 3 \\
\text { mils. }\end{array}$ & $\begin{array}{l}3.0 \\
3.5 \\
4.0 \\
4.5 \\
5.0\end{array}$ & $\begin{array}{l}1439 \pm 3.8 \\
1357 \pm 2.8 \\
1302 \pm 3.5 \\
1202 \pm 3.5 \\
1103 \pm 2.8\end{array}$ & $\begin{array}{l}80.5 \pm 1.4 \\
67.5 \pm 1.4 \\
56.0 \pm 1.4 \\
47.5 \pm 1.4 \\
39.0 \pm 1.4\end{array}$ & ב. & $\begin{array}{r}1358.5 \\
1307.5 \pm 4.1 \\
1246 \pm 3.1 \\
154.5 \pm 3.8 \\
1064 \pm 3.1\end{array}$ & $6.057 \times 10^{5}$ \\
\hline
\end{tabular}

a Manganese has no resonance correction.

foils when covered with the old cadmium covers using the ratio of resonance activities measured with the new and old covers in the second set of measurements.

For the region within $5.0 \mathrm{~cm}$ from the center of the source measurements were initially taken at radii of 4.5 and $5.0 \mathrm{~cm}$ with the first set of foils. The constants in the relation $A_{\mathrm{th}}(r)=C e^{-r^{2 /} r_{0}{ }^{2}}$ were determined from the data and the resulting integral $4 \pi \mathrm{C} \int_{2.1}^{5.0} e^{-r^{2} / r_{0}{ }^{2}} r^{2} d r$ yielded a value of $2.009 \times 10^{7}$ counts $\times \mathrm{cm}^{3} / \mathrm{min}$. Measurements with $1 \mathrm{~cm}^{2}$ manganese foils were extended to a radius of $3 \mathrm{~cm}$ from the source center (table 3), and the extrapolation covers only $0.9 \mathrm{~cm}$ to the aluminum cover around the source. By using the average ratio of the thermal activities of In to $\mathrm{Mn}$ at the radii 4.5 and $5.0 \mathrm{~cm}$ the integral for the manganese foils vields a value of $1.971 \times 10^{7}$ counts $\times \mathrm{cm}^{3} / \mathrm{min}$ for the first set of indium foils (fig. 2). The source has a total nuclear absorption cross section of $0.4 \mathrm{~cm}^{2}$ for thermal neutrons and a correction must be made for selfabsorption. To this end eq (4) may be used to evaluate the flux at the surface of the source and the computed number of neutrons captured internally is $6,000( \pm 30 \%)$ neutrons/sec. For the calculation of $Q$ the following expression was used

$Q=6,000+4 \pi \frac{I_{\mathrm{b}}}{A_{\mathrm{b}}} M_{\mathrm{H}} \frac{\sigma_{\mathrm{H}}}{\sigma_{\mathrm{B}}}\left(1+\epsilon_{\mathrm{ave}}\right) F_{\mathrm{th}} \int_{a}^{\infty} A_{\mathrm{th}} r^{2} d r$.

$I_{\mathrm{b}}$ had increased from 1.761 to $1.767 \times 10^{6}$ disintegrations per sec due to the growth of polonium in the Ra-Be $(\alpha, n)$ sources used in the standard flux when the second set of measurements was taken and

$$
M_{\mathrm{H}}=0.1108 \mathrm{~mole} / \mathrm{cm}^{3} \text {. }
$$

Inserting the data $Q=1.243 \times 10^{6}$ neutrons/sec from the first measurements and $Q=1.270 \times 10^{6}$ neutrons/sec from the second set.

\section{Errors}

Errors from the counting statistics indicated in tables 1 and 2 are small and do not limit the final accuracy. The error in the cam integral is mainly caused by the deviations from the equation of motion and should be less than 1 percent in the second set of measurements where a small correction has been applied. For the region within $5.0 \mathrm{~cm}$ the integral appears good to 2 percent. The self-absorption in the source has been shown to be less than 1 percent by measurements of the activities of a manganese sulfate bath when the source is suspended either alone or in a large carbon block. Exposure in the block reduces the thermal neutron density at the surface of the source by a factor of 3 or 4 thus reducing the selfabsorption. Perturbation of the thermal neutron density by one of the aluminum frames (total volume $0.7 \mathrm{~cm}^{3}$ ) supporting the foil in the tank should be less than 1 percent. In the second set of measurements the 4.5-hour activity of indium from inelastic neutron scattering and gamma excitation was noted and subtracted from the data. This activity was less than 0.5 percent of the initial activity for cadmium covered exposures near the source where the effect was greatest.

For the final result the standard error resulting from compounding the individual errors is 3 percent. The foil data from the second set of measurements has about twice the precision of the first set and the final weighted mean is $Q=1.26_{5} \times 10^{6}( \pm 3 \%)$ neutrons/sec for NBS \#1.

\section{Comparison with Other Standard Sources}

The NBS standard source has been compared with the Argonne and Los Alamos standards by means of intermediary sources. Source \#40 of Los Alamos, a $500 \mathrm{mg} \mathrm{Ra}-\mathrm{Be}(\alpha, n)$ source was compared with the Los Alamos standard in a graphite column and with the NBS source in a $\mathrm{Mn} \mathrm{\textrm {SO } _ { 4 }}$ bath. After resealing in monel the NBS secondary photoneutron standard was compared with the Argonne standard in the subcritical pile. The sourse was then compared with the NBS standard in the $\mathrm{Mn} \mathrm{SO}_{4}$ bath using a bismuthlined proportional counter to measure the tank activities. Table 4 lists the results. Since the errors in comparing the sources with the standards are of the order of 1 percent the errors listed arise principally from the absolute calibrations.

The agreement with Los Alamos is not surprising since the methods are similar, whereas the Argonne calibration involves a measurement of the evolution of helium from boron [9]. 
TABLE 4 .

\begin{tabular}{|c|c|c|c|c|}
\hline \multirow{2}{*}{ Source } & \multirow{2}{*}{ Type } & \multicolumn{2}{|c|}{ Source strength } & \multirow{2}{*}{$\begin{array}{l}\text { Percent- } \\
\text { age devi- } \\
\text { ation from } \\
\text { NBS cali- } \\
\text { bration }\end{array}$} \\
\hline & & NBS calibration & $\begin{array}{c}\text { Comparison cali- } \\
\text { bration }\end{array}$ & \\
\hline $\begin{array}{c}\text { Los Ala- } \\
\text { mos \#40. }\end{array}$ & $\begin{array}{l}\mathrm{Ra}-\mathrm{Be}(\alpha, n) \\
500 \mathrm{mg}\end{array}$ & $\begin{array}{l}5.71 \times 10^{6}( \pm 3 \%) \\
\text { Jan. } 1953\end{array}$ & $\begin{array}{l}5.77 \times 10^{6}( \pm 5 \%) \\
\text { Los Alamos } \\
\text { Nov. } 1952\end{array}$ & $+1.1 \%$ \\
\hline $\mathrm{NBS} \# 2$ & $\begin{array}{l}\text { Ra-Be }(\gamma, n) \\
1.0 \mathrm{~g}\end{array}$ & $\begin{array}{l}1.20 \times 10^{6}( \pm 3 \%) \\
\text { Sept. } 1953\end{array}$ & $\begin{array}{l}1.31 \times 10^{6}( \pm 7 \%) \\
\text { Argonne } \\
\text { March } 1952\end{array}$ & $+9.2 \%$ \\
\hline
\end{tabular}

The authors express their appreciation to $H$. Rosenwasser, R. Olcott, and W. Bailey for assistance in some of the measurements, and to L. Costrell for construction of electronic equipment.

\section{References}

[1] L. F. Curtiss and A. Carson, Phys. Rev. ;6, 1412 (1949).

[2] R. L. Walker, U. S. Atomic Energy Commission Document, MDDC 414 (Oct. 22, 1946).

[3] J. De Juren and H. Rosenwasser, J. Research NBS 52, 93 (1954) RP2477.

[4] Thode, Mac Namara, Lossing, and Collins, J. Am. Chem. Soc. $\mathbf{7 0 ,} 3008$ (1948).

[5] H. L. Anderson, E. Fermi, A. Wattenberg, G. L. Weil, and W. H. Zinn, Pnys. Rev. 72, 16 (1947)

[6] B. Hamermesh, G. Ringo, and S. Wexler, Phys. Rev. 90, 603 (1953)

[7] J. De Juren and H. Rosenwasser, J. Research NBS 51, 203 (1953) RP2452.

[8] C. W. Tittle, Nucleonics 9, 60 (1951); E. D. Klema and R. H. Ritchie, Phys. Rev. 87, 167 (1952).

[9] F. G. P. Seidl and S. P. Harris, Rev. Sci. Instr. 18, 897 (1947).

\section{Appendix - Derivation of Cam Equations}

To determine the motion let: $A_{0}$ be the initial counting rate when the foils reach the final position at $r=20 \mathrm{~cm}, r_{1}$ and $r_{2}$ the initial and final positions of the foils, $T$ the total time of irradiation, and $\lambda$ the foil disintegration constant. Then an increment of activity $d A_{0}=4 \pi K A_{\mathrm{th}}(r) r^{2} d r$ where $K$ is the constant of proportionality to be determined. But $d A_{0}=\lambda A_{\text {th }}(r) e^{-\lambda(T-t)} d t$ where $t$ is the time at which the foil is at $r$. Equating the two expressions for $d A_{0}$

$$
4 \pi K r^{2} d r=\lambda e^{-\lambda(T-t)} d t
$$

and integrating

$$
\frac{4 \pi}{3} K r^{3}=e^{-\lambda(T-t)}+C .
$$

The integration constant is determined from the boundary conditions

$$
\begin{aligned}
t & =0 \text { when } r=r_{1}, \\
t & =T \text { when } r=r_{2}, \\
C & =\frac{4 \pi}{3} K r_{1}^{3}-e^{-\lambda T}, \text { and } \\
C & =\frac{4}{3} \pi K r_{2}^{3}-1 .
\end{aligned}
$$

Solving for $K$ :

$$
K=\frac{3\left(1-e^{-\lambda T}\right)}{4 \pi\left(r_{2}^{3}-r_{1}^{3}\right)}
$$

and since

$$
\int_{r_{1}}^{r_{2}} d A_{0}=A_{0}, \frac{A_{0}}{K}=4 \pi \int_{r_{1}}^{r_{2}} A_{\text {th }}(r) r^{2} d r
$$

Solving for $r$ using $K$ from eq (11)

$$
r=r_{1}\left[1+\left(\frac{r_{2}^{3}}{r_{1}^{3}}-1\right) \frac{\left(e^{\lambda t}-1\right)}{\left(e^{\lambda T}-1\right)}\right]^{1 / 3} .
$$

A synchronous clock motor drives a specially cut cam attached to the top of the tank containing the source. Rotation of the cam causes movement of a follower arm which displaces the foils radially in the tank. Initially the follower arm movement permitted weights to unwind control wires from a drum and displace the suspended foil holders. In the second series of measurements two foil holders $180^{\circ}$ apart were displaced by control wires which were attached directly to the follower arm and rotated on individual pulleys instead of the drum.

The cam was designed for a 2 hour transit time using a value of $54.0 \mathrm{~min}$ for the indium half-life, corresponding to $K=2.382 \times 10^{-5} \mathrm{~cm}^{-3}$. After the initial cutting the curvature of the cam was modified until the movement of the foil in the tank followed $r=f(t)$ in eq (13) within a few tenths of a millimeter as measured with the cathetometer at 5 min interval positions.

Washington, August 4, 1954. 